\title{
Off-label use of thalidomide for the treatment of a bleeding cutaneous metastasis
}

\author{
Federico Silvestri, Andrea Da Porto, Antonella Labombarda, Elisa Mansutti, Adolfo Rogato \\ Division of Internal Medicine, General Hospital, Latisana (UD), Italy
}

\begin{abstract}
We herein describe the case of a 65 -year-old man with frequent hospitalizations for severe anemia due to several recurrent bleedings of a cutaneous metastasis of hepatocellular carcinoma, and its successful off label treatment with thalidomide therapy for controlling bleeding and reducing transfusion requirements.
\end{abstract}

\section{Case Report}

A 65-year-old man was first admitted to our Division in July, 2013, for severe anemia [hemoglobin $(\mathrm{Hb})$ level of $6.5 \mathrm{~g} / \mathrm{dL}]$. Family and personal anamnesis were uneventful, apart from a history of previous alcohol abuse.

In June, 2012, he was diagnosed as a carrier of multifocal hepatocellular carcinoma; the diagnosis was done on the basis of abdominal computed tomography (CT), elevate $\alpha$-fetoprotein serum levels and liver biopsy. The histological examination showed polygonal hepatocytes, displaying an eosinophilic granular cytoplasm, rounded nuclei and prominent nucleoli, with the presence of vascular invasion, the grade of differentiation was grade 3 . The immunohistochemistry was positive for $\alpha$-fetoprotein and negative for cytokeratin 7 and 19. Hepatocellular carcinoma (HCC) staging at baseline was stage B ac-

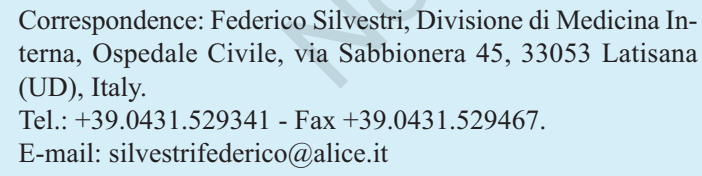

Key words: Thalidomide; off-label; bleeding metastasis.

Conflict of interest: the authors declare no potential conflict of interest.

Received for publication: 7 December 2014.

Revision received: 19 January 2015.

Accepted for publication: 22 January 2015.

This work is licensed under a Creative Commons Attribution NonCommercial 3.0 License (CC BY-NC 3.0).

(C) Copyright F. Silvestri et al., 2015

Licensee PAGEPress, Italy

Italian Journal of Medicine 2015; 9:377-379

doi:10.4081/itjm.2015.570 cording to Barcelona-Clinic Liver Cancer classification. After oncologic evaluation he was then treated with sorafenib from September 2012 to July 2013.

Unfortunately the disease progressed and, at the time of admission to our Division, the patient showed lung, bone and skin metastases. In particular a huge metastasis $(9 \times 7 \mathrm{~cm}$; Figure 1) of the breastbone body was actively bleeding and causing the anemia.

The patient was given tranexamic acid intravenously, locally medicated with pressure medication and submitted to transfusion with 5 units of packed red cells (PRC); he was dismissed with an $\mathrm{Hb}$ level of $11.0 \mathrm{~g} / \mathrm{dL}$.

During the following months he was admitted to our Division other four times (from July to December, 2013), always for severe anemia due to bleeding of the cutaneous metastasis. A total of 36 units of PRC were administered during that period. Figure 2 shows $\mathrm{Hb}$ levels at hospital admission, number of PRC administered and $\mathrm{Hb}$ levels at hospital discharge during these five hospitalizations.

He was also evaluated by a surgeon, in order to verify the feasibility of a surgical approach; this was eventually denied since the CT scan showed the neoplastic tissue/mass infiltrating the breastbone body and approaching the aortic arch (Figure 3).

During the last hospitalization, the off-label use of thalidomide was offered to the patient, in the hypothesis that the antiangiogenic effect of the drug could help to stop bleeding. Informed consensus was signed by patient.

On December 14, 2013, thalidomide, $100 \mathrm{mg}$ daily, was started, without the association of any antithrombotic treatment. Ever since the metastasis did not bleed anymore and the patient maintained stable $\mathrm{Hb}$ levels around 9-9.5 g/dL.

He was followed as an outpatient with a blood test every two weeks for three months; during this period of time he did not experience any adverse effect of thalidomide in particular no thrombotic or hemor- 
rhagic adverse events. Eventually he died of neoplastic cachexia with no evidence of further bleeding.

\section{Discussion}

Thalidomide was first introduced in Europe in 1950s as a treatment for morning sickness, but was withdrawn early due to its severe teratogenic effects. ${ }^{1}$ In the following years thalidomide, for its anti-inflammatory activity, was successfully used in the treatment of multiple diseases including multiple mieloma, ${ }^{2}$ erythema nodosum, ${ }^{3}$ Behçet's disease ${ }^{4}$ and graft versus host disease. ${ }^{5}$

Thalidomide is even known because of some antiangiogenic effects, so that in the last few years it was used for therapy of recurrent gastrointestinal bleeding due to angiodysplasia ${ }^{6}$ as well as refractory Crohn's disease. ${ }^{7}$ No randomized trials are available but several case series have shown an interesting efficacy of thalidomide in reducing bleeding rate and in particular in reducing the need of blood transfusion in treated patients. ${ }^{6,7}$

The antiangiogenic effects of thalidomide seem to be related to a decrease in the synthesis of vascular endothelial growth factor (VEGF) and basic fibroblast growth factor as suggested by in vitro and in vivo studies. $^{7-9}$

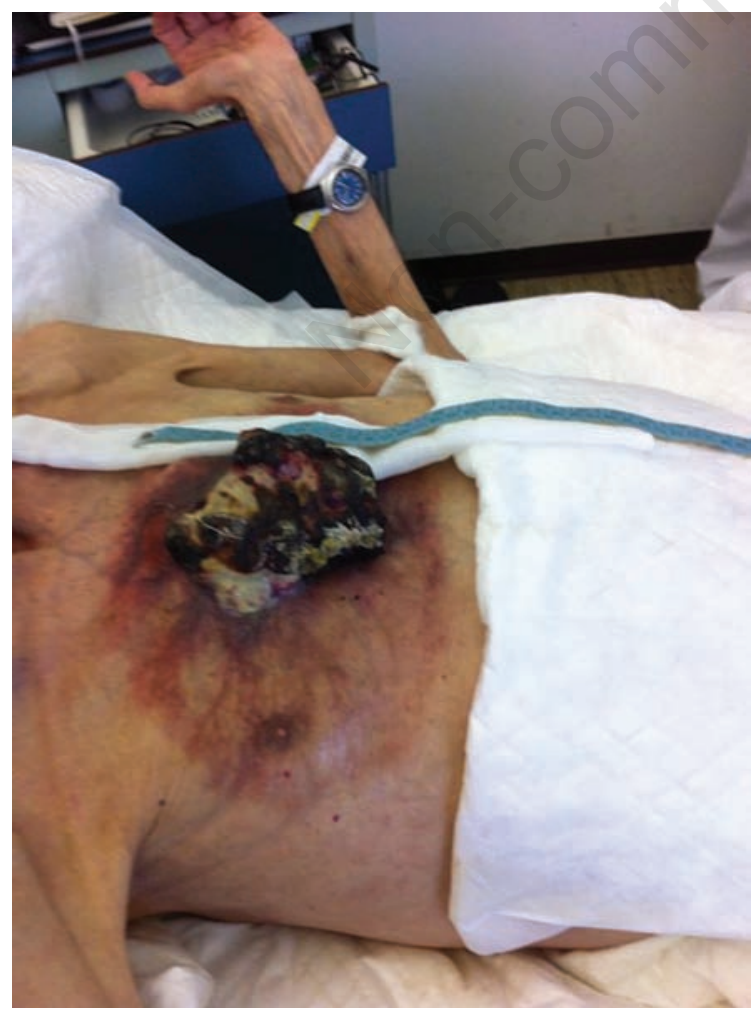

Figure 1. Picture of skin bleeding metastasis.
Hepatocellular carcinoma is a highly vascularized tumor due to the process of angiogenesis and $\mathrm{HCC}$ angiogenesis has been identified as being critical for tumor growth and metastases. VEGF plays a crucial role in the development of metastases in HCC as suggested also in very recent studies, ${ }^{10}$ so it seemed reasonable the palliative-off label use of thalidomide in this patient.

For the lack of randomized controlled trials the recommended dose to prevent bleeding is not established yet. Some Authors recommend a dosage of 300 $\mathrm{mg}$ /day, other 50-100 mg daily. Thalidomide has several side effects including; nausea, constipation, severe painful peripheral neuropathy, dizziness or lighthead-

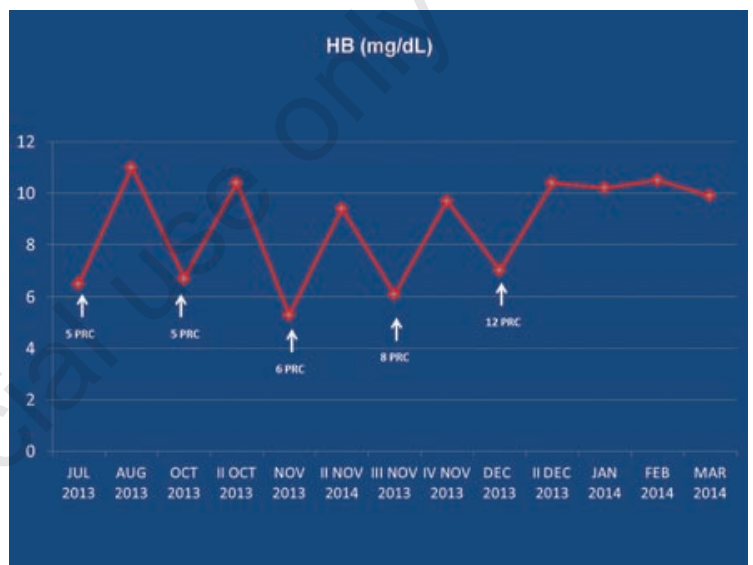

Figure 2. Hemoglobin $(\mathrm{Hb})$ levels at hospital admission, number of packed red cells (PRC) administered and $\mathrm{Hb}$ levels at hospital discharge during these five hospitalizations and during follow-up.

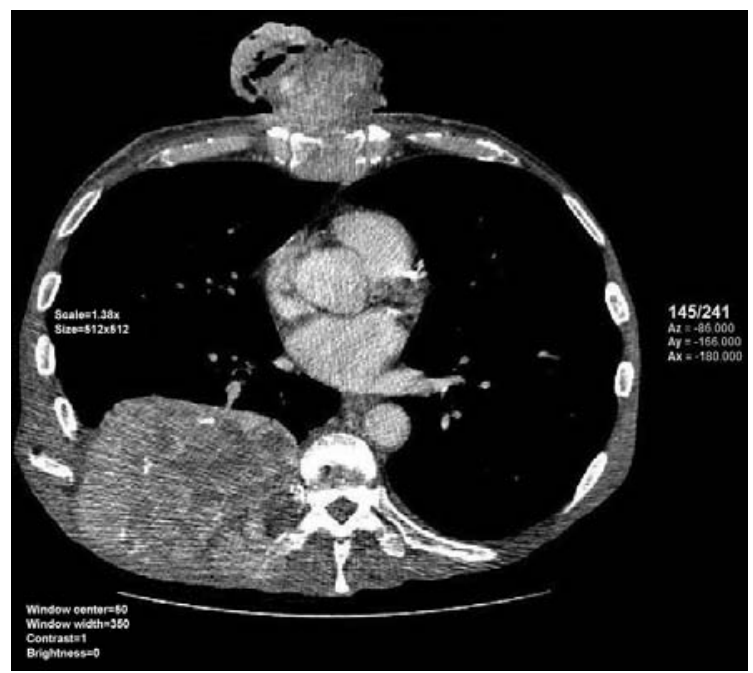

Figure 3. Computed tomography scan image in which is evident the skin metastasis. 
edness, muscle weakness, anxiety and cough that seem to be dose dependent. Therefore to our opinion the starting dose of 50-100 $\mathrm{mg} /$ daily should be safer in minimizing the side effects of the drug.

\section{Conclusions}

To our knowledge this is the first report of thalidomide use to prevent recurrent bleeding in skin metastasis of HCC. Our results suggest a potential off label use of thalidomide in bleeding tumors or metastases in order to reduce transfusion requirements and hospitalization.

\section{References}

1. McBride WG. Thalidomide and congenital abnormalities. Lancet 1961;2:1358.

2. Hicks LK, Kaynes AE, Reece DE, et al. Haematology disease site group of the cancer care Ontario program in evidence based care. A meta-analysis and systematic review of thalidomide for patients with previous untreated multiple myeloma. Cancer Treat Rev 2008;34:442-52.
3. Walker SL, Waters MF, Lockwood DN. The role of thalidomide for treatment of erythema nodosum leprosum. Lepr Rev 2007;78:197-215.

4. Direskeneli H, Ergun T, Yavuz S, et al. Thalidomide has both anti-inflammatory and regulatory effects in Behçet's disease. Clin Rheumatol 2008;27:373-5.

5. Van de Poel MH, Pasman PC, Schouten HC. The use of thalidomide in chronic refractory graft versus host disease. Neth J Med 2001;59:45-9.

6. Kamalaporn P, Saranavan R, Cirocco M, et al. Thalidomide for the treatment of gastrointestinal bleeding from aniodysplasias: a case series. Eur J Gastroenterol Hepatol 2009;21:1347-50.

7. Baudiz J, Schachschal G, Wedel S, Lochs H. Thalidomide for treatment of severe intestinal bleeding. Gut 2004;53:609-12.

8. Kenyon BM, Browne F, D'Amato RJ. Effects of thalidomide and related metabolites in a mouse corneal model of neovascularization. Exp Eye Res 1997;64:971-9.

9. D'Amato RJ, Loughnan MS, Flynn E, et al. Thalidomide in an inhibitor of angiogenesis. Proc Natl Acad Sci U S A 1994;91:4082-5.

10. Liu N, Yang J, Huang Y, et al. Vascular endothelial growth factor accelerates establishment of a model of hepatic metastasis in Walker-256 tumor-bearing rats. Am J Med Sci 2014 [Epub ahead of print]. 